\title{
UNIVERSITYOF
}

FORWARD

THINKING

WESTMINSTER用

WestminsterResearch

http://www.westminster.ac.uk/westminsterresearch

\section{Performance Evaluation of a Statistical and a Neural Network \\ Model for Nonrigid Shape-Based Registration}

Angelopoulou, A., Psarrou, A., Garcia-Rodriguez, J. and

Mentzelopoulos, $\mathbf{M}$.

This is a copy of the author's accepted version of a paper subsequently published in the proceedings of the Sixth International Conference on Image Processing Theory, Tools and Applications (IPTA'16), Finland, 12 to 15 Dec 2016.

It is available online at:

https://dx.doi.org/10.1109/IPTA.2016.7820990

(C) 2016 IEEE . Personal use of this material is permitted. Permission from IEEE must be obtained for all other uses, in any current or future media, including reprinting/republishing this material for advertising or promotional purposes, creating new collective works, for resale or redistribution to servers or lists, or reuse of any copyrighted component of this work in other works.

The WestminsterResearch online digital archive at the University of Westminster aims to make the research output of the University available to a wider audience. Copyright and Moral Rights remain with the authors and/or copyright owners.

Whilst further distribution of specific materials from within this archive is forbidden, you may freely distribute the URL of WestminsterResearch: ((http://westminsterresearch.wmin.ac.uk/)).

In case of abuse or copyright appearing without permission e-mail repository@westminster.ac.uk 


\title{
Performance Evaluation of a Statistical and a Neural Network Model for Nonrigid Shape-Based Registration
}

\author{
Alexandra Psarrou, Anastassia Angelopoulou, Markos Mentzelopoulos, and José García-Rodríguez
}

\begin{abstract}
Shape-based registration methods frequently encounters in the domains of computer vision, image processing and medical imaging. The registration problem is to find an optimal transformation/mapping between sets of rigid or nonrigid objects and to automatically solve for correspondences. In this paper we present a comparison of two different probabilistic methods, the entropy and the growing neural gas network (GNG), as general feature-based registration algorithms. Using entropy shape modelling is performed by connecting the point sets with the highest probability of curvature information, while with GNG the points sets are connected using nearest-neighbour relationships derived from competitive hebbian learning. In order to compare performances we use different levels of shape deformation starting with a simple shape $2 D$ MRI brain ventricles and moving to more complicated shapes like hands. Results both quantitatively and qualitatively are given for both sets.
\end{abstract}

Index Terms-Shape registration, entropy, self-organising networks, probabilistic models

\section{INTRODUCTION}

Over the last decades there has been an increasing interest in using neural networks and computer vision techniques to allow users to directly explore and manipulate objects in a more natural and intuitive environment [1], [2], [3], [4]. With the recent rise of non-intrusive sensors (e.g. Microsofts Kinect, Leap motion) gesture recognition and realtime $3 D$ interaction has added an extra dimension to humanmachine interaction. However, the images captured, which are effectively a $2 D$ projection of a $3 D$ object, can become very complex for any recognition system which will require the correct registration between the shapes. The need for nonrigid shape-based registration occurs in many real world applications. Tasks like registering human brain MRI images in brain mapping, tracking objects in motion, smooth key frame matching in digital animation and $3 D$ morphing in virtual reality applications, all require a robust model not pron to noise that can solve for correct correspondences between a set of shapes.

While a lot of research has been focused on efficient detectors and classifiers, little attention has been paid to efficiently labeling and acquiring suitable training data which

A. Psarrou is with the Computer Vision and Imaging Research Group, Department of Computer Science, University of Westminster, United Kingdom (e-mail: psarroa@wmin.ac.uk).

A. Angelopoulou is with the Cognitive Computing Research Group, Department of Computer Science, University of Westminster, United Kingdom (e-mail: agelopa@wmin.ac.uk).

M. Mentzelopoulos is with the Computer Vision and Imaging Research Group, Department of Computer Science, University of Westminster, United Kingdom (e-mail: mentzem@emin.ac.uk).

J. García-Rodríguez is with the Department of Computer Technology, University of Alicante, Spain (e-mail: jgarcia@dtic.ua.es). then can be used by any recognition system. The collection of training data requires the segmentation and alignment of an observation sequence, which is an ill-conditioned task due to measurement noise and human variation in the observation. Furthermore, it is a time consuming and tedious task. Obtaining a set of training examples automatically is a more difficult task. Existing approaches to minimise the labeling effort [5], [6], [7], [8], [9] use a classifier which is trained in a small number of examples. Then the classifier is applied on a training sequence and the detected patches are added to the previous set of examples. However, in order to learn the model a great amount of hand-labelled images is needed.

Because of the complexity of non-rigid models such as hands and faces, most methods simplify the task and either equally space the point sets along the shape [10] or group the points into higher level structures such as lines, curves or surfaces and parameterising the points along these attributes [11], [12], [13], [14]. An alternative method to the above is to use probabilistic correspondences [15], [16], [17]. With this method the shapes are modeled either linearly by solving a linear optimisation problem such as the least-square problem or nonlinearly by configuring correspondences in non-linear manifolds [18], [19], [20], [21], [22], [23], [24].

In this work, we propose to compare the efficiency in establishing correspondences of two different probabilistic models: the entropy model for extracting points with high entropy values derived by the probability of being curvature points or not, and the GNG model where points are represented as neurons randomly generated in a highdimensional space and which the adaptation of the network is based on nearest-neighbour relationships and local error measurements.

The remaining of the paper is organised as follows. Section 2 provides a detailed description of the entropy and the different thresholds required to characterise the contour of the ventricles by selecting regions of high curvature. Section 3 provides a description of the topology learning algorithm GNG. A set of experimental results along with qualitative analysis is presented in Section 4, before we conclude in Section 5.

\section{ENTROPY}

The fundamental premise of information theory is that the generation of information can be modeled as a probabilistic process that can be measured in a manner that agrees with intuition [25]. Entropy defines the average amount of information obtained by observing a single source output. As 
the entropy magnitude increases, more uncertainty and thus more information is associated with the source. If the source symbols are equally probable, the entropy of uncertainty (Equation 4) is maximized and the source provides the greatest possible average information per source symbol. Entropy is a a good way of representing the impurity or unpredictability of a set of data since it is dependent on the context in which the measurement is taken. An approach to describe parts of an image in terms of varying scales in space using the entropy is discussed in [26], [27].

In this paper we use entropy as a statistical metric between high curvature points. For each ventricle the entropy is calculated not as a global feature for the entire ventricle image but as local operator. We consider that if we distribute the entropy among the ventricle curvature edges, then the regions (points) that contain the salient information for the ventricle shape representation will contain the higher levels of distribution. Therefore any change in the object shape of one of this salient regions it will affect its relevant semantic information in the entire image. Calculating the entropy of our model involves two stages: 1) Extract the curvature points and 2) calculate the entropy of these points.

\section{A. Curvature points}

Curvature is the rate of change in the slope of an image. To obtain if a point in the image is a salient curvature point we are calculating the differences of the angle orientation between neighbouring edge points. As we are moving across the boundaries in a clockwise direction, a curvature point $\rho$ exists if the change in the slope is above a certain range (Figure 1). In our case the edge point $\rho$ is a curvature point if the angle of change exceeds $15^{\circ}$. Because the interpolation depends on the length of the individual segments relative to the overall length of the boundary, the distance between the consecutive edge points must be sufficient big enough in order to be able to detect a change in the slope.

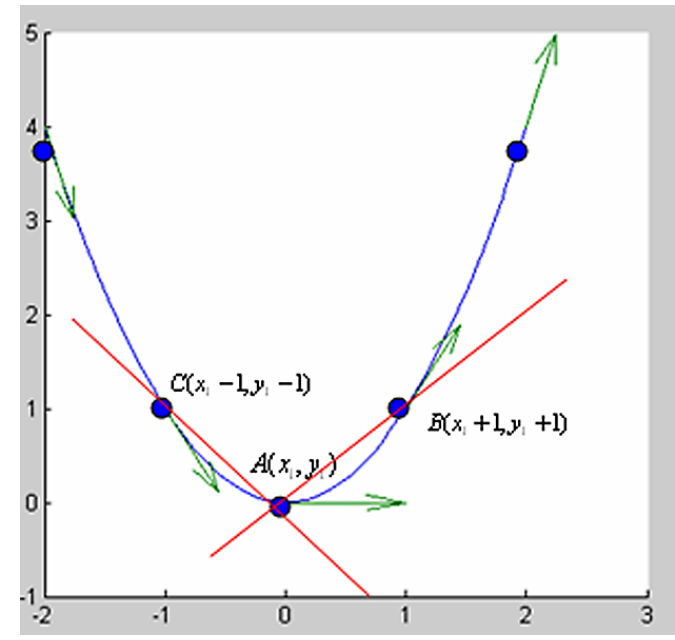

Fig. 1. Example of curvature. Points A,B,C are all edge points for the current shape. The current examined point $\mathrm{A}$ with coordinates $x_{1}, y_{1}$ will be a curvature point because the angle of change $C \hat{A} B$ between the orientation vectors (green vectors) is greater than $15^{\circ}$.

\section{B. Entropy Algorithm}

Our proposed model is working as a local operator with respect to the entire ventricle and hand topology. Therefore what we try to extract is not only whether a point is a curvature edge point but also its importance in the entire shape. To define its importance we produce a histogram of entropies over the range of the points (eg: $\mathrm{M}=270$ ) (Figure 2). Knowing the curvature points which may differ from shape to shape the probability of this curvature point across the entire data set is given by:

$$
p_{f}(k)=h_{f}(k) /(M x N)
$$

where $k$ is one of the points of the image $(1 \leq k \leq 270)$ and $h_{f}(k)$ is the frequency of the $k^{t h}$ point in the dataset and can be estimated as:

$$
h_{f}(k)=\frac{1}{M x N} \sum_{i=1}^{M} k_{i}
$$

$\mathrm{M}$ is the number of points in the data set and $\mathrm{N}$ the number of images. Then the information quantity $Q_{f}(k)$ transmitted by this curvature point is equal to the $\log _{2}$ of the inverse probability of curvature points $\left(p_{f}(k)\right)$ :

$$
Q_{f}(k)=\log _{2}\left(\frac{1}{p_{f}(k)}\right)=-\log _{2}\left(p_{f}(k)\right)
$$

The entropy $E$ generated by the source for each of the image points is determined from the multiplication result between the above information $Q_{f}(k)$ and its probability of being a curvature point.

$$
E_{f}(k)=p_{f}(k) \times Q_{f}(k)
$$

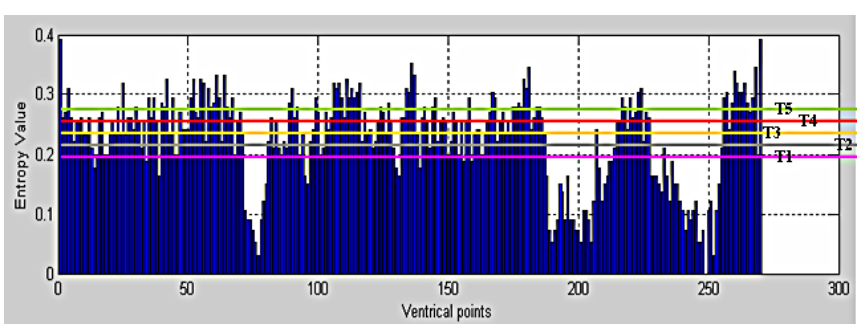

Fig. 2. Entropy Histogram. Number of points for each ventricle (same number of points in all images=M) versus their corresponding curvature entropy among the dataset $\sum_{\text {ventricle }=1}^{N} E_{f}(k)$.

Figure 2 shows that the highest the probability of the $k^{\text {th }}$ curvature point the highest the entropy at this point which means that identical points will correspond across the data set. After calculating the entropy distribution we kept thresholds with significant difference in the resulting points. The different thresholds are: $\mathrm{T} 1=0.2$ (188 points), T2=0.22 (169 points), T3=0.24 (144 points), T4=0.26 (100 points) $\&$ T5= 0.28 (64 points). 


\section{Growing NEURAL GAS (GNG)}

The Growing Neural Gas (GNG) [28] is an incremental neural model able to learn the topological relations of a given set of input patterns by means of competitive hebbian learning. In [18] we used GNG as a training process to map the dimensionality of the input space to a chosen reduced latent topology. In this work we use it as a comparative measurement to the entropy. While entropy use curvature information to extract points of interest GNG uses neighbouring relations to adapt the map to the contour and distribute the neurons. However, neurons are inserted in areas with the highest accumulated error. In both models shape variations are taken by varying the first six principal components and restricting the variance to $\beta_{i}\{ \pm 3 \sigma\}$.

The GNG algorithm consists of the following:

- A set $A$ of cluster centres known as nodes. Each node $c \in N$ has its associated reference vector $\left\{x_{c}\right\}_{c=1}^{N} \in \mathbb{R}^{q}$. The reference vectors indicate the nodes' position or receptive field centre in the input distribution. The nodes move towards the input distribution by adapting their position to the input's geometry using a winner take all mapping. Generating $\xi_{w}$ input signals from the random vector $W$, we want to find a mapping $G: \mathbb{R}^{q} \longrightarrow \mathbb{R}^{A}$ and its inverse $F: \mathbb{R}^{A} \longrightarrow \mathbb{R}^{q}$ such that $\forall c=1, \ldots,|N|$,

$$
\begin{gathered}
f(x)=E_{W \mid g(W)}\{W \mid g(W)=x\}, \forall x \in\left\{x_{c}\right\}_{c=1}^{N} \subseteq \mathbb{R}^{q} \\
g(W)=\arg \min _{v \in\left\{x_{c}\right\}_{c=1}^{N}}\left\|W-x_{v}\right\|
\end{gathered}
$$

where $E$ is the distance operator of the data points from the random vector $W$ projecting onto $f(x), g(W)$ is the projection operator, $\left\{x_{c}\right\}_{c=1}^{N} \subseteq \mathbb{R}^{q}$ are the reference vectors of the network and $x_{v}$ is the winner node. $q$ and $A$ denote the dimensionality of the input space and the reduced latent topology. Figure 3 shows an example of a $2 D$ GNG network with its associated Voronoi diagram in $2 D$ discrete distribution.

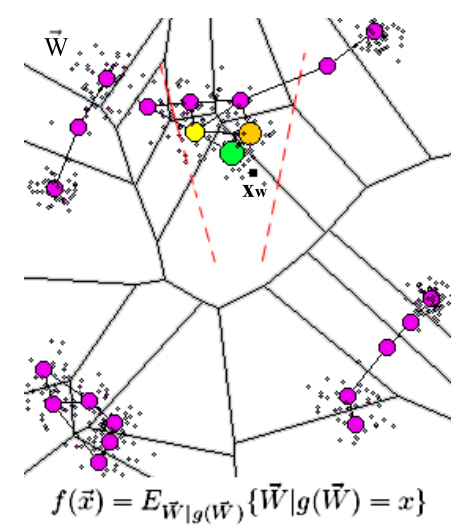

Fig. 3. A random signal $\xi_{w}$ on the discrete input distribution and the best matching within the topological neighbourhood of $\left\{x_{c}\right\}_{c=1}^{N} \subseteq \mathbb{R}^{q}$. In this example, the green node is the winner node of the network among its direct topological neighbours (orange and yellow nodes). The orange node is the second nearest node to the random signal $\xi_{w}$.
- Local accumulated error measurements and insertion of nodes. Each node $c \in N$ with its associated reference vector $\left\{x_{c}\right\}_{c=1}^{N} \in \mathbb{R}^{q}$ has an error variable $E_{x_{c}}$ which is updated at every iteration according to:

$$
\Delta E_{x_{v}}=\left\|\xi_{w}-x_{v}\right\|^{2}
$$

The local accumulated error is a statistical measure and is used for the insertion and the distribution of new nodes. Nodes with larger errors will cover greater area of the input probability distribution, since their distance from the generated signal is updated by the squared distance. Knowing where the error is large, if the number of the associated reference vectors belonging to the input space is an integer multiple of a parameter $\lambda$, a new node $x_{r}$ is inserted halfway between the node with the largest local accumulated error $x_{q}$ and its neighbour $x_{f}$.

$$
x_{r}=\frac{x_{q}+x_{f}}{2}
$$

All connections are updated and local errors are decreased by:

$$
\begin{aligned}
& \Delta E_{x_{q}}=-\alpha E_{x_{q}} \\
& \Delta E_{x_{f}}=-\alpha E_{x_{f}}
\end{aligned}
$$

A global decrease according to:

$$
\Delta E_{x_{c}}=-\beta E_{x_{c}}
$$

is performed to all local errors by a constant $\beta$. This is important since new errors will gain greater influence in the network resulting in a better representation of the topology.

- A set $C$ of edges (connections) between pair of nodes. These connections are not weighted and its purpose is to define the topological structure. The edges are determined using the competitive hebbian learning method. The updating rule of the algorithm is expressed as:

$$
\begin{gathered}
\Delta x_{v}=\varepsilon_{x}\left(\xi_{w}-x_{v}\right) \\
\Delta x_{c}=\varepsilon_{n}\left(\xi_{w}-x_{c}\right), \forall c \in N
\end{gathered}
$$

where $\varepsilon_{x}$ and $\varepsilon_{n}$ represent the constant learning rates for the winner node $x_{v}$ and its topological neighbours $x_{c}$. An edge aging scheme is used to remove connections that are invalid due to the activation of the node during the adaptation process. Thus, the network topology is modified by removing edges not being refreshed by a time interval $\alpha_{\max }$ and subsequently by removing the nodes connected to these edges.

\section{EXPERIMENTS}

In this section we represent the experimental setup for performance evaluation and comparison of the entropy method with the GNG and the equally spaced points. All methods have been developed and tested on a desktop machine of $2.26 \mathrm{GHz}$ Pentium IV processor. These methods have been implemented in MATLAB. The execution time for all three methods is $2.22(\mathrm{sec})$ for $\mathrm{GNG}, 1.82(\mathrm{sec})$ for entropy and $2.44(\mathrm{sec})$ for equally spaced. 


\section{A. The Training Sets}

We used two sets of data with varying difficulty in the shape deformation. For the ventricles the data were obtained from the MNI BIC Centre for Imaging at McGill University, Canada. These images are $1 \mathrm{~mm}$ thick, $181 \times 217$ pixels per slice, $3 \%$ noise and $20 \%$ INU. We used a total of 36 images in this study, 16 were derived from the MNI BIC dataset and 20 were generated using the first principal component of the MNI BIC images. For the hands we have recorded hand images from 5 participants. To create this data set we have recorded images over several days and a simple webcam was used with image resolution $800 \times 600$. In total, we have recorded over 12000 frames, and for computational efficiency, we have resized the images from each set to $300 \times 225,200 \times 160,198 \times 234$, and $124 \times 123$ pixels. We obtained the data set from the University of Alicante, Spain and the University of Westminster, UK. Also, we tested our method with 49 images from Mikkel B. Stegmann ${ }^{1}$ online data set. In total we have run the experiments on a data set of 174 images.

\section{B. The Results}

Figure 4 and 5 show qualitative results of the extracted points and the maps obtained by the entropy and the GNG. The results show that both GNG and entropy lead to correct extraction of anatomical shapes when the correct topology preservation of the map is achieved or an adequate entropy value is chosen.
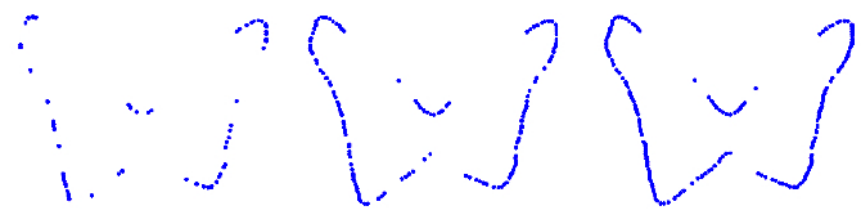

Image A
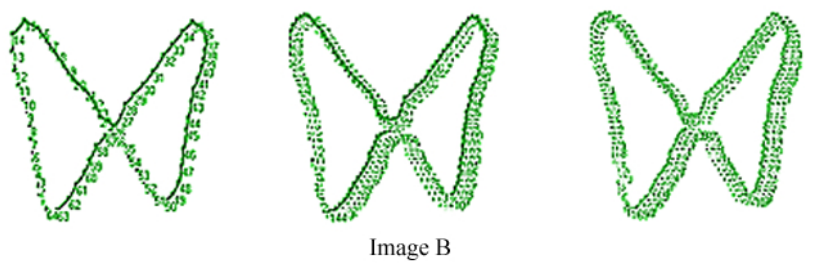

Fig. 4. Image A shows the points extracted by entropy with thresholds T5 (64 points), T3 (144 points) and T1 (188 points). Image B shows the network size of 64,144 and 188 neurons.

It is interesting to note that whilst there is significant difference between 64, and 188 points -not enough points to represent the object and overfitting- the mapping with 144 is sufficient enough in both methods to reconstruct the shapes. The reason is that for the current size of the images the distance between the points is short enough so adding extra points does not give more accuracy in placement. A good adaptation with 144 points takes 14 (entropy) and 11 (GNG) seconds approximately.

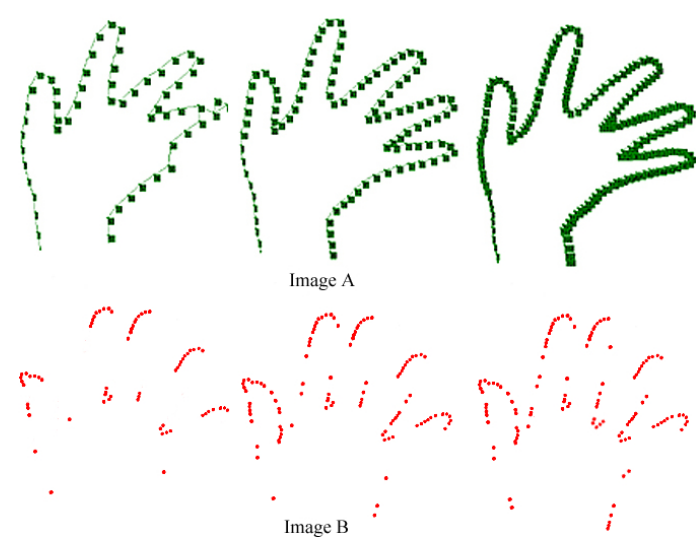

Fig. 5. Image A shows the topologies of different network sizes. Image B shows the entropy points.

In Figure 6 the mode of variation for the equally spaced and entropy points are displayed by varying the first shape parameter $\beta_{i}\{ \pm 3 \sigma\}$ over the training set. The results qualitatively indicate that entropy leads to correct extraction of corners (sharp and smooth) of anatomical shapes and gives better reconstruction of the original shape when is backprojected from the PCA space (Image A from Figure 7) [18]. Image B from Figure 7 shows a back-projection to the original by taking all the points for both equally and entropy methods. Since the entropy points were selected from the equally spaced the shapes overlap and yield an accurate approximation of the original image by selecting the first six principal components.
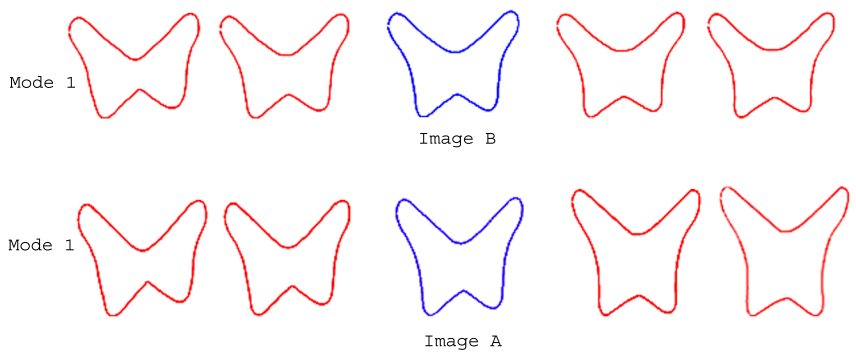

Fig. 6. The first mode $(m=1)$ of variation for the equally spaced (Image B) and entropy points (Image A). Range of variation $-3 \sqrt{\lambda} \leq \beta_{i} \leq 3 \sqrt{\lambda}$.

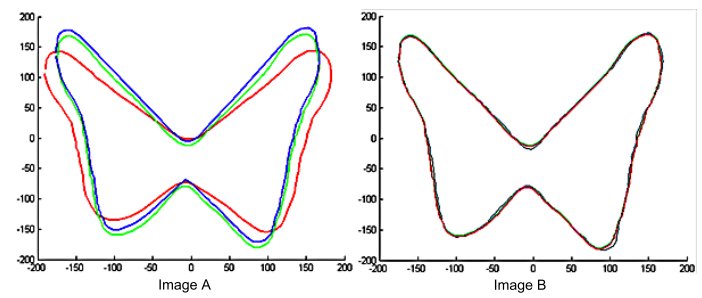

Fig. 7. Back-projection to the original (blue colour) by selecting all the points from the images and by taking the first six principal components. Image A: The superposition of both the equally spaced (red colour) and the entropy (green colour) back-projected when 147 points are used. Image B: The same as A but in this case when all the 270 points are used. 


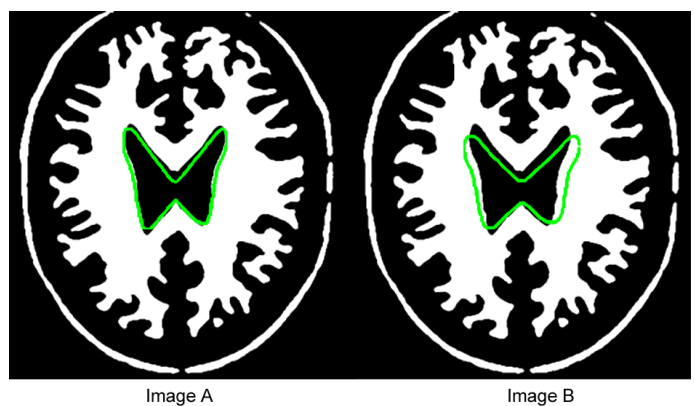

Fig. 8. Superimposed shape instances to the training set.

In Figure 8 one shape variation from the two different methods was superimposed to the original image from the training set. The entropy method (Image A) effectively captures the variability of the training set and present only valid shape instances while the equally spaced method (Image B) is overfitted and expands over the boundary of the original image. Figure 9 shows the first six principal components used to capture the $95 \%$ of the training set. From the left hand side diagram one can see that the first two principal components of the entropy capture higher variations since their eigenvalues are greater compared to the equally spaced thus giving greater variability to the magnitude of the eigenvectors.
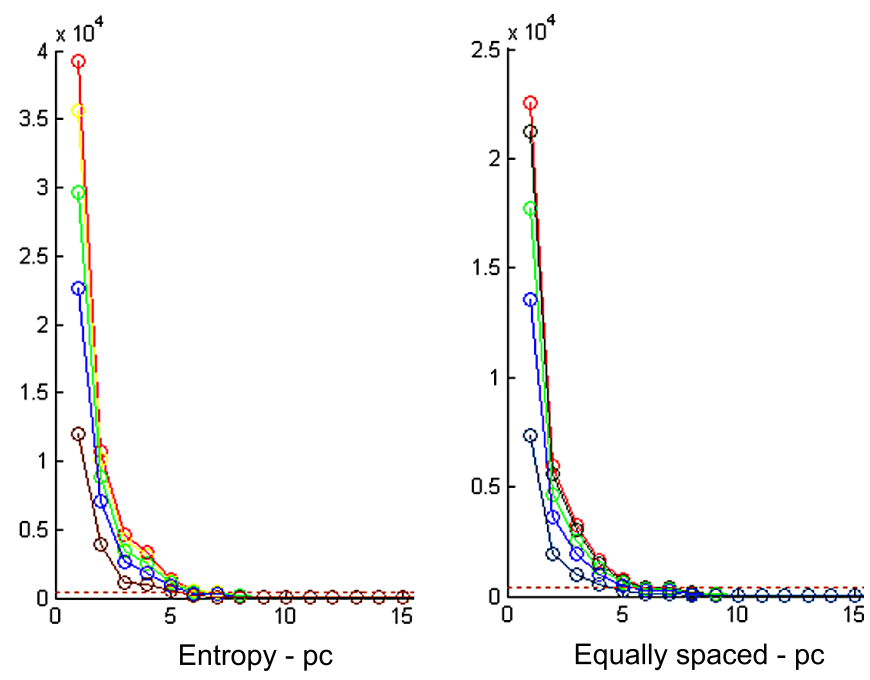

Fig. 9. The first six principal components of entropy and equally spaced.

Figure 10 shows a comparative diagram of the entropy taken at different thresholds (T1, T2, T3, T4, T5) and the equivalent GNG points by calculating the Mean Squared Error (MSE) between the original shape and the backprojected from the PCA space. The diagram shows that as the number of entropy points increases (point 188 from the diagram) the error minimises. On the contrary the error difference between the two methods increases as the number of points decreases (point 64 from the diagram). This is explained by the fact that entropy is performed only on the curvature points while the error in GNG is measured in the whole contour and since a few neurons are used to represent the map the topology is lost.

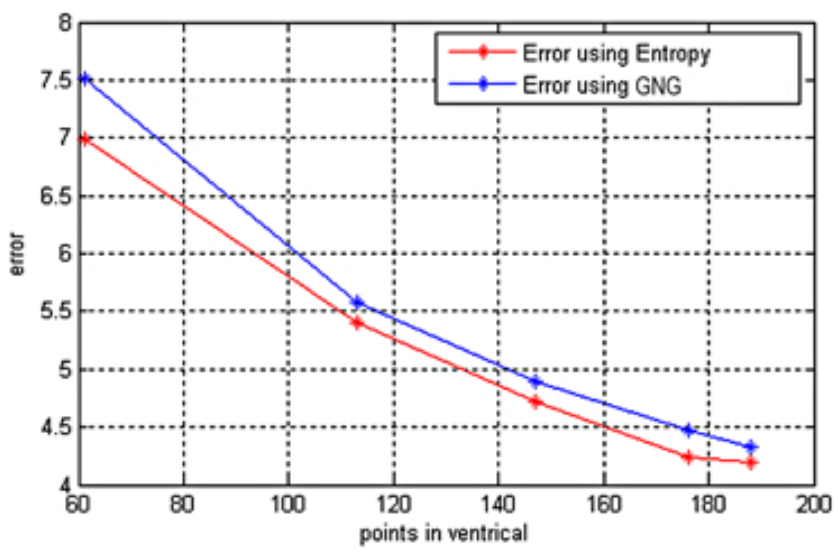

Fig. 10. Error Measurement used for both methods.

Although entropy performs better compared to the equally spaced method, with the GNG algorithm performs slightly better where fewer points are selected.

\section{Conclusions}

This paper has presented a performance evaluation of entropy, GNG and equally spaced points for point set extraction algorithms for non-rigid objects that can be used in shape modelling. We have compared the reconstruction error for contour extraction algorithms using points that were selected a) from a growing neural gas network with nearest-neighbour relations, b) evenly-spaced and c) from entropy based on the highest probability of the curvature point. As expected the reconstruction error of the contour is the least when all the points (270) on the contours are used. However, when fewer points are used the entropy produces slightly better qualitatively (legal shapes) and quantitatively (smaller reconstruction error) shapes. Practically we have shown that the optimum number of points (144 points) required to represent the contour depends mainly on the resolution of the input space and if it is not sufficient then the topology preservation is lost. In future work, we would investigate the generalisation of the methods to 3D models. Likewise, the acceleration of the whole system should be completed on GPUs.

\section{REFERENCES}

[1] H. Koike, Y. Sato, and Y. Kobayashi, "Integrating Paper and Digital Information on Enhanced Desk: A Method for Real Time Finger Tracking on an Augmented Desk System," ACM Transactions on Computer-Human Interaction, vol. 8, no. 4, pp. 307-322, 2001.

[2] H. Tamimi, H. Andreasson, A. Treptow, T. Duckett, and A. Zell, "Localization of mobile with omnidirectional vision using particle filter and iterative sift," Robotics and Autonomous Systems, vol. 54, no. 9, pp. 758-765, 2006.

[3] R. Zhou, Y. Junsong, M. Jingjing, and Z. Zhengyou, "Robust partbased hand gesture recognition using kinect sensor," IEEE Transactions on Multimedia, vol. 15, no. 5, pp. 1110-1120, 2013.

[4] W. Guile and K. Wenxiong, "Robust fingertip detection in a complex environment," IEEE Transactions on Multimedia, vol. 18, no. 6, pp. 978-987, 2016. 
[5] M.W. Lee and R. Nevatia, "Integrating Component Cues for Human Pose Estimation," In Proc. of the IEEE International Workshop on Visual Surveillance and Performance Evaluation of Tracking and Surveillance, pp. 41-48, 2005.

[6] A. Levin, P. Viola, and Y. Freund, "Unsupervised improvement of visual detectors using co-training," In Proc. of the IEEE International Conference on Computer Vision, vol. I, pp. 626-633, 2003.

[7] V. Nair and J. Clark, "An Unsupervised, Online Learning Framework for Moving Object Detection," In Proc. of the IEEE Conference on Computer Vision and Pattern Recongition, vol. II, pp. 317-324, 2004.

[8] J. Sivic, M. Everingham, and A. Zisserman, "Person Spotting: Video Shot Retrieval for Face Sets," In International Conference on Image and Video Retrieval, pp. 226-236, 2005.

[9] C. Cuevas, E.M. Yanez, and N. Garca, "Tool for semiautomatic labeling of moving objects in video sequences: Tslab," Sensors, vol. 15 , no. 7, pp. 15159-15178, 2015.

[10] A. Baumberg and D. Hogg, "Learning Flexible Models from Image Sequences," In Proc. of the $3^{\text {rd }}$ European Conference on Computer Vision, vol. 1, pp. 299-308, 1994.

[11] G. L. Scott and H. C. Longuet-Higgins, "An algorithm for associating the features of two images," In Proc. of the Royal Society of London, vol. B244, pp. 21-26, 1991.

[12] S. Scalroff and A. Pentland, "Modal Matching for Correspondence and Recognition," IEEE Trans. on Pattern Aanalysis and Machine Intelligence, vol. 17, no. 6, pp. 545-561, 1995.

[13] A. Rangarajan, H. Chui, and F. L. Bookstein, "The softassign procrustes matching algorithm," In Proc. of the $15^{\text {th }}$ Conference on Information Processing in Medical Imaging, pp. 29-42, 1997.

[14] S. Belongie, J. Malik, and J. Puzicha, "Matching Shapes," In Proc. of the $8^{\text {th }}$ Internationl Conference on Computer Vision, vol. 1, pp. 454-463, 2001.

[15] F. Wang, J. B. Vemuri, and A. Rangarajan, "Simultaneous Nonrigid Registration of Multiple Point Sets and Atlas Construction," In Proc. of the $9^{\text {th }}$ European Conference on Computer Vision, ECCV 2006, LNCS 3953, pp. 551-563, 2006.

[16] K. Chang and J. Ghosh, "A Unified Model for Probabilistic Principal Surfaces," IEEE Trans. on Pattern Aanalysis and Machine Intelligence, vol. 23, no. 1, pp. 22-41, 2001.

[17] H. Chui and A. Rangarajan, "A new point matching algorithm for non-rigid registration," In Proc. of Computer Vision and Image Understanding (CVIU), vol. 89, pp. 114-141, 2003.

[18] A. Angelopoulou, J. García, and A. Psarrou, "Learning 2D Hand Shapes Using the Topology Preservation Model GNG," In Proc. of the $9^{\text {th }}$ European Conference on Computer Vision, ECCV 2006, LNCS 3951, pp. 313-324, 2006.

[19] G. Gupta, A. Psarrou, A. Angelopoulou, and J. García, "Region analysis through close contour transformation using growing neural gas," In Proc. of the International Joint Conference on Neural Networks, IJCNN2012, pp. 1-8, 2012.

[20] E. Stergiopoulou and N. Papamarkos, "Hand gesture recognition using a neural network shape fitting technique," Engineering Applications of Artificial Intelligence, vol. 22, no. 8, pp. 1141-1158, 2009.

[21] C. Sui, Appearance-based hand gesture identification, Master of Engineering, University of New South Wales, 2011.

[22] R.L.M. Rêgo, A.F.R. Araújo, and F.B. de Lima Neto, "Growing SelfOrganizing Maps for Surface Reconstruction from Unstructured Point Clouds," In Proc. of the International Joint Conference on Artificial Neural Networks, IJCNN'07, pp. 1900 - 1905, 2007.

[23] A.M. Cretu, E.M. Petriu, and P. Payeur, "Evaluation of growing neural gas networks for selective 3D scanning," In Proc. of IEEE International Workshop on Robotics and Sensors Environments, pp. $108-113,2008$.

[24] Y. Holdstein and A. Fischer, "Three-dimensional surface reconstruction using meshing growing neural gas (MGNG)," The Visual Computer: International Journal of Computer Graphics, vol. 24, no. 4, pp. 295-302, 2008.

[25] R. C. Gonzalez and R. E. Woods, Digital Image Processing, Prentice Hall, 2002.

[26] T. Kadir and M. Brady, "Scale, saliency and image description," IJCV, vol. 45, no. 2, pp. 83-105, November 2001.

[27] M. Mentzelopoulos and A. Psarrou, "Key-frame Extraction Algorithm Using Entropy Difference," In Proc. of the 6th ACM SIGMMA International Workshop on Multimedia Information Retrieval (MIR 04), pp. 39-45, 2004.
[28] B. Fritzke, "A growing Neural Gas Network Learns Topologies," In Advances in Neural Information Processing Systems 7 (NIPS'94), pp. 625-632, 1995 